\title{
AN ANALOGUE OF THE SCHWARZ LEMMA FOR BOUNDED SYMMETRIC DOMAINS
}

\author{
GIANCARLO TRAVAGLINI
}

\begin{abstract}
We determine the best possible estimate for the determinant of the Jacobian of a holomorphic mapping of a bounded symmetric domain into a ball.
\end{abstract}

1. This note is concerned with the following analogue of the classical Schwarz lemma. Suppose $D=\prod_{\mu} D_{\mu}$ is a bounded symmetric domain in $\mathbf{C}^{n}$ with irreducible components $D_{\mu}$, realized as a circular starlike bounded domain with center 0 , in accordance with Harish-Chandra's imbedding (here and in the sequel the reference for the theory of bounded symmetric domains is [5]). Let $F: D \rightarrow B_{n}$ be a holomorphic mapping of $D$ into the unit ball of $C^{n}$. Let $J(F)$ denote the Jacobian matrix of $F$. To estimate $\operatorname{det}(J(F)(z))$ we can suppose $F(0)=0$ and evaluate the Jacobian at the origin (we write $J(F)(0)=J(F)$ ). Let $l_{\mu}$ and $n_{\mu}$ denote the rank and the dimension of $D_{\mu}$ respectively. We prove that

$$
|\operatorname{det}(J(F))| \leqslant n^{-n / 2} \cdot \prod_{\mu}\left(n_{\mu} / l_{\mu}\right)^{n_{\mu} / 2},
$$

and that this estimate cannot be improved.

The above inequality was proved by Caratheodory [2] for the polydisc and by Kubota [7] for the classical Cartan domains. Related results may be found in [3] under more general hypotheses, but in our case these results do not give sharp estimates. Let us refer also to Korányi [4] for a different, and more classical, extension of the Schwarz lemma to bounded symmetric domains.

2. Let $D_{\mu}=G_{\mu} / K_{\mu}$, where $G_{\mu}$ is the connected component of the group of holomorphic automorphisms of $D_{\mu}$ and $K_{\mu}$ is the subgroup of $G_{\mu}$ which leaves 0 fixed ( $K_{\mu}$ is a connected compact group of unitary transformations). Let $\mathrm{g}_{\mu}^{\mathbf{C}}$ and $\mathfrak{f}_{\mu}^{\mathbf{C}}$ be the complexifications of the Lie algebras of $G_{\mu}$ and $K_{\mu}$ respectively. Under the symmetry $\sigma_{\mu}$ of $\mathfrak{g}_{\mu}$ we have the decomposition $\mathfrak{g}_{\mu}=\mathfrak{f}_{\mu}+\mathfrak{p}_{\mu}$ into eigenspaces of $\sigma_{\mu}$ for the eigenvalues +1 and -1 respectively. We choose a Cartan subalgebra $\mathfrak{h}_{\mu}$ in $\mathfrak{f}_{\mu}$ : then $\mathfrak{h}_{\mu}^{\mathbf{C}}$ is a Cartan subalgebra in $\mathfrak{g}_{\mu}^{\mathbf{C}}$. To every noncompact root $\alpha_{\mu}$ (a root of $\mathfrak{g}_{\mu}^{\mathbf{C}}$ which is not a root of $f_{\mu}^{C}$ ) we associate in the standard way the element $E_{\alpha}^{\mu}$ of $\mathfrak{g}_{\mu}^{\mathrm{C}}$. The canonical realization of $D_{\mu}$ is in the complex vector space $\mathfrak{p}_{\mu}^{-}$which is the subalgebra of $g_{\mu}^{\mathbf{C}}$ spanned by the $E_{-\alpha}\left(\alpha\right.$ positive noncompact). Let $\Delta_{\mu}$ denote the Harish-Chandra

Received by the editors June 1, 1982.

1980 Mathematics Subject Classification. Primary $32 \mathrm{H} 99$.

Key words and phrases. Schwarz lemma, bounded symmetric domains.

${ }^{1}$ Research supported in part by C.N.R. 
system of strongly orthogonal noncompact positive roots. It is known that $a_{\mu}=$ $\Sigma_{\alpha_{\mu} \in \Delta_{\mu}} \mathbf{R}\left(E_{\alpha}^{\mu}+E_{-\alpha}^{\mu}\right)$ is a maximal abelian subalgebra contained in $\mathfrak{p}_{\mu}$. Let $A_{\mu}=$ exp $a_{\mu}$. Since $G_{\mu}=K_{\mu} A_{\mu} K_{\mu}$ we have $D_{\mu}=K_{\mu} A_{\mu} \cdot 0$, and the orbit $A_{\mu} \cdot 0$ is a unit ( $l_{\mu}$-dimensional) cube around 0 .

We shall use the above notation with $\mu$ missed when we shall refer to $D$.

3. We state the theorem.

TheOREM. Let $D=\prod_{\mu} D_{\mu}$ be a bounded symmetric domain in $\mathbf{C}^{n}$, with irreducible components $D_{\mu}$, in the standard realization. Let $F$ be a holomorphic mapping of $D$ into the unit ball $B_{n}$ in $\mathbf{C}^{n}$ such that $F(0)=0$. Then (writing $J(F)(0)=J(F)$ )

$$
|\operatorname{det}(J(F))| \leqslant n^{-n / 2} \cdot \prod_{\mu}\left(n_{\mu} / l_{\mu}\right)^{n_{\mu}}
$$

( $n_{\mu}$ and $l_{\mu}$ are the dimension and the rank of $D_{\mu}$ respectively). Moreover, there exists a mapping $\tilde{F}: D \rightarrow B_{n}$ for which the equality holds.

Proof. Fix a vertex $E^{\mu}$ of the unit cube $A_{\mu} \cdot 0$ contained in $D_{\mu}$. Let $S_{\mu}=K_{\mu} \cdot E^{\mu}$ denote the Silov boundary of $D_{\mu}\left(K_{\mu}\right.$ acts transitively on $\left.S_{\mu}\right)$. We put $E_{1}^{\mu}=E^{\mu} \cdot l_{\mu}^{-1 / 2}$ and we choose an orthonormal basis $\left\{E_{i}^{\mu}\right\}_{i=1}^{n_{\underline{\mu}}}$ in $D_{\mu}$. Let $z_{1}^{(\mu)}, \ldots, z_{n_{\mu}}^{(\mu)}$ be the coordinate functions associated to this basis.

Now let $z_{i}^{(\mu)}$ and $z_{j}^{(\gamma)}$ be two coordinate functions on the domains $D_{\mu}$ and $D_{\gamma}$ respectively. We denote by $S$ the Silov boundary of $D$ and we have (for $\mu \neq \gamma$ )

$$
\int_{S} z_{i}^{(\mu)} \overline{z_{j}^{(\gamma)}}=\int_{S_{\mu}} z_{i}^{(\mu)} \cdot \int_{S_{\gamma}} \overline{z_{j}^{(\gamma)}}=0 \quad(\mu \neq \gamma) .
$$

For $\mu=\gamma$ we write $z_{i}$ in place of $z_{i}^{(\mu)}$ and we have

$$
\int_{S} z_{i} \overline{z_{j}}=\int_{S_{\mu}} z_{i} \overline{z_{j}}=\int_{K_{\mu}} z_{i}\left(k \cdot E^{\mu}\right) \cdot \overline{z_{j}\left(k \cdot E^{\mu}\right)} d k .
$$

But

$$
\begin{aligned}
z_{i}\left(k \cdot E^{\mu}\right) & =\left(k \cdot E^{\mu}, E_{i}^{\mu}\right)=l_{\mu}^{1 / 2} \cdot\left(k \cdot E_{1}^{\mu}, E_{i}^{\mu}\right) \\
& =l_{\mu}^{1 / 2} \cdot \operatorname{Ad}_{\mathfrak{p}_{\mu}^{-}}(k)_{1, i}
\end{aligned}
$$

where $\operatorname{Ad}_{\mathfrak{p}_{\mu}^{-}}(k)_{1, i}$ is the $(1, i)$-coefficient of the adjoint representation of $K_{\mu}$ acting on the complex space $\mathfrak{p}_{\mu}^{-}$in which $D_{\mu}$ is realized. Since $D_{\mu}$ is irreducible, the $n_{\mu}$-dimensional unitary representation $\mathrm{Ad}_{\mathfrak{p}_{\mu}^{-}}$is irreducible. Then (1), (2), (3) and Schur's lemma give

$$
\begin{aligned}
\int_{S} z_{i}^{(\mu)} \overline{z_{j}^{(\gamma)}} & =\delta_{\mu, \gamma} \cdot l_{\mu} \int_{K_{\mu}} \operatorname{Ad}_{\mathfrak{p}_{\mu}^{-}}(k)_{1, i} \cdot \overline{\operatorname{Ad}_{\mathfrak{p}_{\mu}^{-}}(k)_{1, j}} d k \\
& =\delta_{\mu, \gamma} \cdot \delta_{i, j} \cdot l_{\mu} / n_{\mu} \quad(\text { Kronecker's } \delta) .
\end{aligned}
$$

We now observe that if $P_{\sigma}$ and $P_{\nu}$ are homogeneous polynomials on $\mathfrak{p}^{-}$with degree $\sigma$ and $\nu$ respectively, then

$$
\int_{S} P_{\sigma} \overline{P_{\nu}}=0 \quad(\text { if } \sigma \neq \nu)
$$


To prove (5) we can use the so-called Bochner's Trick (see $[1,6])$. First, we recall that $K$ contains all the elements of the form $e^{i \theta} I$, where $\theta$ is any real number and $I$ is the identity operator. Then, by homogeneity,

$$
\begin{aligned}
\int_{S} P_{\sigma} \overline{P_{\nu}} & =\frac{1}{2 \pi} \int_{S} \int_{0}^{2 \pi} P_{\sigma}\left(e^{i \theta} z\right) \cdot \overline{P_{\nu}\left(e^{i \theta} z\right)} d s d \theta \\
& =\frac{1}{2 \pi} \int_{S} \int_{0}^{2 \pi} e^{i(\sigma-\nu) \theta} \cdot P_{\sigma}(z) \cdot \overline{P_{\nu}(z)} d s d \theta=0 \quad(\text { if } \sigma \neq \nu) .
\end{aligned}
$$

Now, let $F$ be as in the statement of the Theorem. We write $F=\left(f_{1}, \ldots, f_{n}\right)$ and $f_{i}\left(z_{1}, \ldots, z_{n}\right)=a_{1}^{i} z_{1}+\cdots+a_{n}^{i} z_{n}+$ (higher terms), $i=1, \ldots, n$. The almost everywhere defined boundary values of the bounded holomorphic mapping $F$ will also be denoted by $F$. Thus

$$
1 \geqslant \int_{S}|F|^{2}=\sum_{i=1}^{n} \int_{S}\left|f_{i}\right|^{2}
$$

We develop each $f_{i}$ in homogeneous polynomials and we use the orthogonality relations (4) and (5) to obtain

$$
1 \geqslant \sum_{i=1}^{n} \int_{S} \mid a_{1}^{i} z_{1}+\cdots+a_{n}^{i} z_{n}+\left.(\text { higher terms })\right|^{2} \geqslant \sum_{i=1}^{n}\left(l_{\mu_{t}} / n_{\mu_{t}}\right) \cdot\left|a_{j}^{i}\right|^{2}
$$

where $D_{\mu_{i}}$ is the domain on which $z_{i}$ is defined.

Now let $H$ be the $n \times n$ matrix with coefficients $h_{j}^{i}=\left(l_{\mu_{t}} / n_{\mu_{t}}\right)^{1 / 2} \cdot a_{j}^{i}$. Then

$$
\operatorname{trace}\left(H H^{*}\right)=\sum_{i, j=1}^{n}\left(l_{\mu_{i}} / n_{\mu_{i}}\right) \cdot\left|a_{j}^{i}\right|^{2}
$$

Here $H H^{*}$ is a positive definite Hermitian matrix. Hence, it is unitarily equivalent to a diagonal matrix $V$ with strictly positive entries on the diagonal. Then

$$
\operatorname{Trace}\left(H H^{*}\right)=\text { Trace } V \geqslant n(\operatorname{det}(V))^{1 / n}=n\left(\operatorname{det} H H^{*}\right)^{1 / n} .
$$

Finally, since $\operatorname{det}\left(H H^{*}\right)=\prod_{\mu}\left(l_{\mu} / n_{\mu}\right)^{n_{\mu}} \cdot\left|\operatorname{det}\left(a_{j}^{i}\right)\right|^{1 / 2}$, we get from (6), (7) and (8):

$$
\begin{aligned}
|\operatorname{det} J(F)| & =\left|\operatorname{det}\left(a_{j}^{i}\right)\right|=\prod_{\mu}\left(n_{\mu} / l_{\mu}\right)^{n_{\mu} / 2} \cdot\left|\operatorname{det}\left(H H^{*}\right)\right|^{1 / 2} \\
& \leqslant n^{-n / 2} \cdot \prod_{\mu}\left(n_{\mu} / l_{\mu}\right)^{n_{\mu} / 2} .
\end{aligned}
$$

We conclude the proof by getting a mapping $\tilde{F}$ for which the equality holds in (9). Let $\tilde{F}: D \rightarrow \mathbf{C}^{n}$ be such that $F=\left(\tilde{f}_{1}, \ldots, \tilde{f}_{n}\right)$, where, for $i=1, \ldots, n$,

$$
\tilde{f}_{i}\left(z_{1}, \ldots, z_{n}\right)=n^{-1 / 2} \cdot\left(n_{\mu} / l_{\mu}\right)^{1 / 2} \cdot z_{i}
$$

where $D_{\mu}$ is the irreducible domain on which the coordinate function $z_{i}$ was defined. Observe that $D_{\mu}=K_{\mu} A_{\mu} \cdot 0$ is contained in an $n_{\mu}$-dimensional ball of radius $l_{\mu}^{-1 / 2}$. Hence $\left.\tilde{F}\right|_{D_{\mu}}\left(D_{\mu}\right)$ is contained in a ball of radius $\left(n_{\mu} / n\right)^{1 / 2}$. Hence $\tilde{F}(D)$ is contained in a ball of radius $\left(n^{-1} \cdot \Sigma_{\mu} n_{\mu}\right)^{1 / 2}=1$, i.e. $\tilde{F}(D) \subseteq B_{n}$. Now, clearly,

$$
|\operatorname{det}(J(\tilde{F}))|=n^{-n / 2} \cdot \prod_{\mu}\left(n_{\mu} / l_{\mu}\right)^{n_{\mu} / 2}
$$

and the proof is complete. 
REMARK. The orthogonality relation (5) becomes unnecessary if we show that $F$ may be chosen linear without loss of generality. This is not hard to prove. Indeed a standard application of the classical Schwarz lemma shows that the following holds. Let

$$
\begin{aligned}
F=\left(a_{1}^{i} z_{1}+\cdots+a_{n}^{1} z_{n}+\right. & \text { (higher terms) } \\
& +\cdots+a_{1}^{n} z_{1}+\cdots+a_{n}^{n} z_{n}+\text { (higher terms)) }
\end{aligned}
$$

be a mapping from the bounded symmetric domain $D$ into $B_{n}$; then also the linear map

$$
F_{\text {lin }}=\left(a_{1}^{1} z_{1}+\cdots+a_{n}^{1} z_{n}, \ldots, a_{1}^{n} z_{1}+\cdots+a_{n}^{n} z_{n}\right)
$$

sends $D$ into $B_{n}$.

AcKnowledgement. The author is grateful to Professor Adam Korányi for several helpful suggestions concerning this paper. Moreover, the author wishes to thank the Department of Mathematics of Washington University in St. Louis for friendship and hospitality.

ADDED IN PROOF. A similar result has been independently obtained by Y. Kubota in a paper to appear in Bull. London Math. Soc.

\section{REFERENCES}

1. S. Bochner, Classes of holomorphic functions of several variables in circular domains, Proc. Nat. Acad. Sci. U. S. A. 46 (1960), 721-723.

2. C. Carathéodory, Über die Abbildungen, die durch systeme von analytischen funktionen von mehreren Veränderlichen erzeugt werden, Math. Z. 34 (1932), 758-792.

3. S. Kobayashi, Hyperbolic manifolds and holomorphic mappings, Dekker, New York, 1970.

4. A. Korányi, A Schwarz lemma for bounded symmetric domains, Proc. Amer. Math. Soc. 17 (1966), 210-213.

5. , Holomorphic and harmonic functions on bounded symmetric domains, CIME Summer Course on Geometry of Bounded Homogeneous Domains, Cremonese, Roma, 1968, pp. 125-197.

6. , Function theory on bounded symmetric domains, Several Complex Variables, Scuola Norm. Sup. Pisa, 1978, pp. 201-216.

7. Y. Kubota, An extremal problem on the classical Cartan domains, Kodai Math. J. 4 (1981), 278-287. Istituto Matematico dell'Universitá, Via Saldini 50, 20133 Milano, Italy (Current address)

Department of Mathematics, Washington University, St. Louis, Missouri 63130 